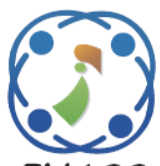

\title{
Computer Assisted Bone Age Estimation of Children Using Middle Finger and Carpal Bones
}

\author{
Aye Aye Aung ${ }^{1 *} \quad$ Zin Mar Win ${ }^{1}$ \\ ${ }^{1}$ University of Computer Studies, Mandalay, Myanmar \\ * Corresponding author's Email: ayeayeaung@ucsm.edu.mm
}

\begin{abstract}
Bone age assessment methods have been replaced from manual to automatic evaluation in medical field. Bone age is also needed for pediatricians and endocrinologists to compare chronological age for growth disorders and endocrinological problems. Recently, bone age estimation has gained remarkable ground in academia and medicine. Automated bone age assessment (BAA) methods are needed to improve the accuracy of the bone age because manual BAA methods are time-consuming and inefficient. Hand radiograph is the most widely applied to calculate the bone age of children. There are several challenges in bone age estimation such as the less of accuracy to segment region of interest (ROI), to identify boundaries and to classify bone age. The aim of the proposed system is to improve robustness and accuracy of the BAA system. The system applies Histogram of Oriented Gradient (HOG) features for middle finger and $\mathrm{Hu}$ moments features for carpal bones. Then, the system uses Support Vector Machines (SVM) by applying extracted features from middle finger and carpal bones for bone age classification. The proposed system uses the dataset from University of Southern California. Combination of HOG features from Epiphysis/Metaphysis region of interests (EMROIs) and Hu moments features from carpal region of interest (CROI) achieved better results than other methods. Running time of combining HOG and $\mathrm{Hu}$ moments features is faster than other methods and this method reduces feature dimensions than using HOG features from EMROIs and CROI. As the experimental results, the accuracy of the system achieves $89 \%$ of Asian (ASI) and African American (BLK) female radiographs and $86 \%$ of ASI and BLK male radiographs in age range from one year to ten years old. In the experiment, this method obtains 1.2717 years of Mean Absolute Error (MAE) compared with radiologist 1 and 1.1997 years of MAE compared with radiologist 2 in testing ASI and BLK females. Then, the system also obtains 1.3681 years of MAE compared with radiologist 1 and 1.3311 years of MAE compared with radiologist 2 in testing ASI and BLK males.
\end{abstract}

Keywords: Bone age assessment, Epiphysis/Metaphysis, Carpal, Hu moments, Support vector machine.

\section{Introduction}

Skeletal maturity estimation is an assessment using left-hand radiographs to analyse the progress of ossification of the hand wrist bones. In several countries worldwide, endocrinological problems in adolescents are already apparent, varying in size and intensity of different age and sexes. Changes in people's diets and eating patterns can lead to endocrine disorders, raising the requirement for a system to predict these problems well in advance [1]. BAA is an essential process to diagnose and treat growth disorder of children. The development of bone age is a significant indicator for children's growth. Hand radiograph has been widely applied to assess skeletal maturity for pediatrics. The difference between the chronological age and bone age may indicate bone ossification abnormalities [2].

BAA is often carried out in pediatric endocrinology, orthodontics and pediatric orthopedics. Bone age is an important predictor for adult height estimation and diagnosing various diseases. The purpose of assessing bone age is to evaluate growth and development and to diagnose and manage pediatric disorders. The accuracy of the estimation of bone age is therefore very important [3]. There is radius, ulna, seven carpals, five metacarpals and fourteen phalanges bones in the hand wrist. Pediatricians and endocrinologists require to 
compare the bone age with chronological age to diagnose diseases with stature disorder in child [4]. Bone age calculation also need in absent birth records. In our part of the world, the absence of birth data is a major issue. Sixty five percent of all births are not registered at five years old in South Asia. The need for a reliable age estimation exists in cases where a child's age needs to be reliable, such as law suits, immigration and professional sports. Bone age is applied in these situations to provide the best measurement for chronological age [4]. The bones of children mature at different rates. Using the discrepancy between chronological age and the bone age, pediatricians will diagnose children's growth disorders, endocrine diseases and diabetes. Hormone imbalance, diabetes or genetics can affect skeletal bones of the children. Therefore, skeletal maturity assessment plays an important role in pediatric radiology.

In bone age estimation, hand wrist $\mathrm{x}$-ray images are suitable because the hand wrist comprises several bones and it is easy to take radiograph of the hand. In bone age calculation, the use of left-hand $\mathrm{x}$-rays rather than the right hand is because most people are right handed, so the right hand is more likely injured than left [3]. Some researchers used features extracted only from Epiphysis/Metaphysis ROIs (EMROIs) or carpal ROI (CROI) that are not accurate for a complete BAA evaluation because they do not facilitate bone growth assessment. The bone age cannot be measured using hand $\mathrm{x}$-rays over eighteen years old because the hand is matured at eighteen years. Therefore, the clavicle is applied to measure the age from 18 to 22 years old [4]. Carpal bones were reliable for BAA from zero to six years old of the children in medical study. For children above 6 years, EMROIs features were very reliable and for age below 5 or 7 years, the EMROI analysis was failed in feature extraction in some cases. In the bone age evaluation, the examination of only EMROI or CROI may not be sufficient, as a minor difference in the shape of these bones may cause a change in the evaluation of bone age. For these reasons, it is required to apply the automated BAA by integrating EMROI and CROI.

BAA is generally performed with hand wrist radiographs by using either Tanner \& Whitehouse (TW) method or Greulich \& Pyle (GP) method. GP method is an atlas matching technique, while the TW method is a score assigning technique. GP is faster and easier than TW method. TW is a more reliable calculation than the GP method and is more relevant in automated BAA.

In this research, the proposed system uses the features extracted from Epiphysis/Metaphysis ROIs
(EMROIs) of middle finger and Carpal ROI (CROI) which is reliable and accurate for a complete bone age estimation. The system applies Support Vector Machine (SVM) classifier with polynomial kernel to assess bone age. The system has five steps such as pre-processing, ROIs segmentation, background subtraction, feature extraction and classification. In the next section, state of research, material and methods, experimental results and conclusion are included.

\section{State of research}

The purpose of this system is to assess automated BAA system using EMROIs from middle finger and CROI from wrist bones. Therefore, we study about existing BAA methods. Several investigators have attempted computerized BAA systems with the advent of digital image processing techniques. Bone age estimation is not a new topic in medical science. In the Roman Empire, the second molar eruption was applied as an indicator to call adolescent male for military service[5]. Age was estimated using tooth eruption to detect a child' age by dentists since nineteenth century[5]. Rontgen discovered estimation of age using $\mathrm{x}$-rays of skeleton [5]. Angerer stated that bone age estimation can be used the carpus bone of the hand in young people in 1886 [6].

Ewa Pietka, Lotfi Kaabi, M. L. Kuo, and H. K. Huang[7] applied carpal bones to determine the bone age. To distinguish the hand from the background, the CROI was defined using a thresholding method. Then, to distinguish between the bones and the soft tissue, a dynamic thresholding approach was applied. Then, mathematical morphology was used to eliminate bones such as radius, ulna, and metacarpals that overlap the borders of carpal bones. Eight features were considered for each carpal bone. Among these 8 features, area, perimeter, the number of carpal bones and ratio 2 are important and then compactness ratio, lengthening ratio, ratio1 and convexity coefficient are not significant.

In 1994, G.K Manos, A.Y. Cairn, I.W. Rickets and D. Sinclair proposed computer-based the segmentation technique of hand radiographs. In this system, the segmentation technique was based on the region growing and region merging. They applied heuristic rules to label either background or bone based on grey level properties in the bone extraction. Lastly, by segmenting the bone outlines, conjugated bones were identified. The segmented bones could be applied as regions of interest in bone age determination using TW2 method [8]. 
A. Gertych, A. Zhang, J. Sayre, S. P. Kurkowska and H.K. Huang [9] developed a computer-aideddiagnosis (CAD) method using carpal bones from new-born to seven years old. This system applied fuzzy classification to classify bone age by using extracted features from carpal bones. They used 205 hand radiographs for bone age determination. Then, it found that the carpal bones are very accurate up to 7 years old in bone age assessment. In [2], D. Giordano, C. Spampinato, G. Scarciofalo and R. Leonardi proposed automated BAA using EMROI and CROI in age range from zero to ten years. They used different of Gaussian filter for EMROI analysis. They applied active contour models and derivative difference of Gaussian filter for CROI extraction. This paper achieved accuracy rate about $87 \%$ on testing 30 hand radiographs. The accuracy of the system was degraded nearly 50\% in applying Trapezium and Trapezoid. They achieve the accuracy of $91 \%$ without considering these bones.

J. Liu, J. Qi, Z. Liu, Q. Ning and X. Luo [10] introduced Particle Swarm Optimization (PSO) to segment bones and extract features, and then applied artificial neural networks (ANN) models. To extract features according to TW3 method, 13 RUS (radius, ulna and short finger bones) regions and 7 carpal bones were applied. Based on Particle Swarm Optimization, 5 features such as scale, morphological characteristics and fusional / adjacent stage of each ROI were applied and input into ANN classifiers. Then, the extracted features were trained with backpropagation algorithm. They applied $1046 \mathrm{x}$-ray images randomly half to train ANNs and the remainder for BAA. Their findings showed that the particle swarm optimization method was efficient to segment and extract features.

J. Seok, B. Hyun, J. K. Vubu and A. Girard [11] developed bone age assessment system by applying hand radiographs. This bone age determination system applied Scale Invariant Feature Transform for feature extraction from hand radiographs and then proposed a Singular Value Decomposition for feature vectors composition. This system trained Neural Network classifier with feature vectors.

In 2016, G. E. Guraksin, H. Uguz and O. K. Baykan[12] developed a BAA system of the left-hand wrist radiographs. The system was performed using C4.5 algorithms, Support Vector Machine, Naive Bayes and k-nearest neighbour to classify bone age. The system used the age from zero to six years of 195 $\mathrm{x}$-ray images. This system extracted 9 features from distal epiphysis of the radius and carpal bones. In this research, support vector machine achieved $72.82 \%$ which has a better performance than other methods.
In 2018, J. A. Choi, Y. C. Kim, S. J. Min and E. K. Khil [13] developed a BAA system called capitohamate $(\mathrm{CH})$ planimetry. This study included 391 children from age range 1 to 180 months. In this system, they applied the area of capitate and hamate of carpal bones from left and right hands radiograph and then compared with GP method. The summation of capitate area and hamate area was used in this study. Experimental result of this system showed an accuracy rate of $84 \%$ in using 782 hand images.

F. Dehghani, A. Karimian and M. Sirous [14] developed BAA system from zero to eighteen years old. They applied scale invariant feature transform, histogram of oriented gradients and local binary pattern methods for feature extraction from EMROI and CROI. Then, they used support vector machine (SVM) and 5 folds cross validation to classify bone age. They applied 442 x-ray images and they achieved the accuracy of $73.88 \%$ for female and $68.63 \%$ for male. In 2020, D. Wang, K. Zhang, J. Ding, and L. Wang[15] proposed Anatomical LocalAware Network (ALA-Net) for automatic bone age estimation. The authors learn the hand structure and extract local information. This model can detect the anatomical ROIs and estimate bone age. Their experimental result obtains MAE value 3.91 on the public RSNA dataset. H. Lee, S. Tajmir, J. Lee, M. Zissen, B. A. Yeshiwas, T. K. Alkasab, G. Choy and S. Do [16]developed automated deep learning system to segment ROI, standardize and pre-process the radiographs and perform BAA. This system used an ImageNet pretrained convolutional neural network (CNN). The performance of this system achieved $61.40 \%$ in male radiographs and $57.32 \%$ in female radiographs in testing age range from 5 to 18 years. As a limitation, this system excluded age range from 0 to 4 years. V. Pinchi, F. D. Luca, M. Focardi, F. Pradella, G. Vitale, F. Ricciardi and G.A. Norelli[17] proposed bone age estimation by combining skeletal and dental age. This system applied Greulich and Pyle's (GP), Tanner-Whitehouse (TW3), Willems' (W) and the Demirjian's (D) methods to estimate skeletal and dental age. D and TW3 combination achieved accuracy rate $83.3 \%$ in female radiographs and $80.4 \%$ in male radiographs in testing age range from 6 to 17 years.

\section{Material and methods}

The proposed bone age estimation system consists of five steps: pre-processing, ROIs segmentation, background subtraction, feature extraction and classification steps. In the preprocessing step, the hand image is enhanced using image filtering method in order to reduce image' 


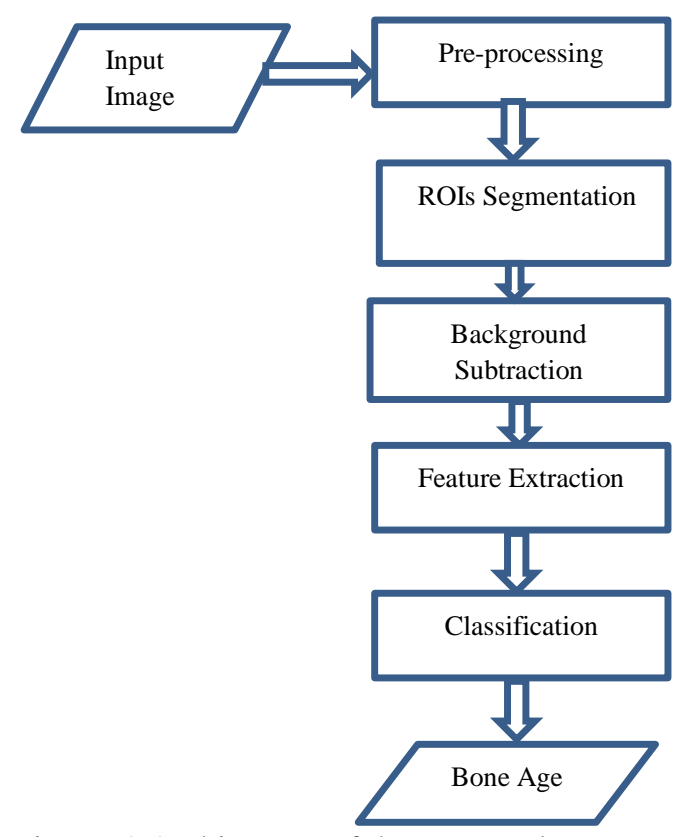

Figure. 1 Architecture of the proposed system

noise. Then, the system segments EMROIs from middle finger and CROI from hand wrist in ROIs segmentation step. The proposed research applies a background subtraction method for segmented ROIs and then extracts features from these segmented ROIs. Finally, the research work applies support vector machine (SVM) classifier for BAA by using features. Fig. 1 shows the architecture of the proposed system.

\subsection{Pre-processing step}

In this step, the system applies anisotropic diffusion method to reduce noises of $x$-ray images. Anisotropic diffusion is an image noise reduction technique without removing significant parts of the image. It removes noises from hand $\mathrm{x}$-ray image without blurring edges. It combines original image with a filter that depends on the original image.

$$
\frac{\partial I}{\partial t}=\nabla c \cdot \nabla I+c(x, y, t) \Delta I
$$

where I is grey scale image, $\nabla$ represents the gradient operator, $\Delta$ is the Laplacian operator and $c(x, y, t)$ is

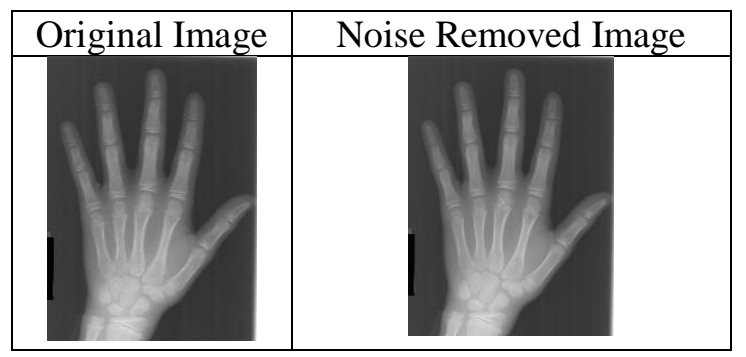

Figure. 2 Original hand image and noise removed image diffusion coefficient [18].The original hand x-rays and noise removed hand $\mathrm{x}$-rays are described in Fig. 2.

\subsection{Region of interests (ROIs) segmentation}

In regions of interests (ROIs) segmentation, the proposed research extracts EMROIs from middle finger and CROI from left hand wrist. The system extracts three EMROIs from middle finger and carpal bones, distal radius and ulna from carpal area. ROIs extraction is carried out according to[19]. The proposed system uses middle finger to extract EMROI segments. The middle finger is extracted using wedge functions on the threshold image. These functions are used to separate each finger and locate each finger's tips, middle and base points by evaluating the local maxima and minima of the wedge function. It forms a binary matrix that is transformed into a set of step-wedge functions. Each of the function corresponds to one row of the matrix. The elements of this function are zeros in the background and ones in the object. The high value refers to the phalangeal area, while the low value refers to the distance between the phalanges. According to the peak number of these functions, the system finds the finger tips, width and the finger region is then extracted. The proposed system applies a filter on grey levels long axis of extracted middle finger to reduce noise and then the first order derivative is applied to search local maxima. These local maxima values represent the bone borders and are used to extract EMROIs. In CROI analysis, carpal bones are extracted from the entire hand by using wedge functions. By detecting the soft tissue junction between thumb and the second finger, the top-right point of the CROI is located. Starting from this point, the system finds the leftmost point of this row. The system defines a rectangle using these two points, in

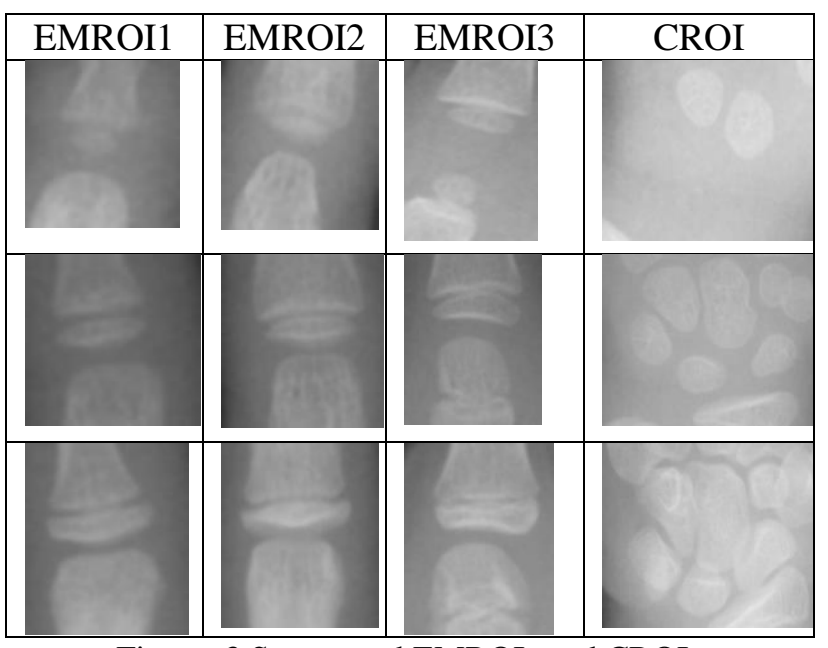

Figure. 3 Segmented EMROIs and CROIs 
which this rectangle identifies CROI [19]. Fig. 3 shows the segmented EMROIs and CROI of hand images.

\subsection{Background subtraction}

Top hat transform technique is applied for background subtraction. To correct the image' nonuniform brightness, this approach is applied. It is used to remove objects through the application of a structural element from the image. To subtract the background from the extracted ROIs, a disk-shaped structuring element with radius $(r=50)$ is used. This technique is the difference between input image and its opening with a structuring element.

$$
T_{w}=f-f o b
$$

where $f$ is grayscale image and $b$ is structuring element of grayscale image and $o$ denotes opening operation and $T_{w}$ is top hat transform image $[14,20]$. Background subtracted ROIs are described in Fig. 4.

After background subtraction, Otsu's thresholding is applied to perform automatic image thresholding. It separates foreground and background pixels. Then, the output is used for the next stage.

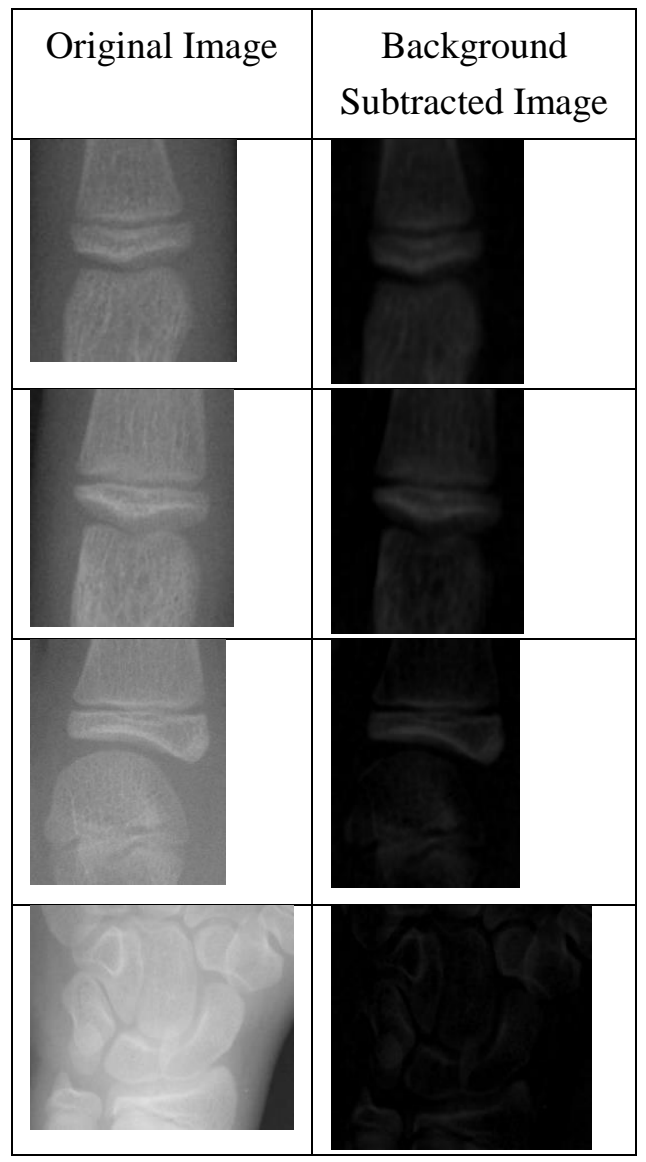

Figure. 4 Background subtracted images

\subsection{Feature extraction}

In feature extraction, Histogram of Oriented Gradient (HOG) and Hu moments methods are used to extract features from the EMROIs of middle finger and CROI of hand wrist. The proposed research uses Histogram of Oriented Gradient (HOG) features extracted from middle finger bones and $\mathrm{Hu}$ moments features extracted from carpal bones. HOG features descriptor focuses on the structure or the shape of an object. HOG method extracts the gradient and orientation of the edges and this method is able to provide the edge direction as well. To define, characterize, and measure the shape of an object in an image, the $\mathrm{Hu}$ moments can be used. Hu moments have been extensively employed as global features in image processing. The number of features obtained are 5292 features of HOG from middle finger and 7 features of $\mathrm{Hu}$ moments from carpal bones. After the concatenation of the feature vectors obtained from $\mathrm{HOG}$ and $\mathrm{Hu}$ moments methods, the total features vector of 5299 is used for classification.

\subsubsection{Histogram of oriented gradient (HOG)}

HOG feature is the local shape information described by the intensity gradients or edge direction. The image is split into a rectangle cell and determines magnitude and orientation of the gradient for a particular cell. Histogram of discretized orientations is constructed in the orientation binning. This step accounts for gradient orientation occurrences in localized portions of the image. To calculate a HOG descriptor, the horizontal gradients and vertical gradients are firstly calculated. After all, the histogram of gradients is calculated. This is simply achieved by filtering the image using the kernels below,

\begin{tabular}{|l|l|l|}
\hline-1 & 0 & 1 \\
\hline
\end{tabular}

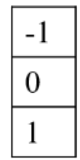

Then, find the magnitude and direction of the gradient using the following formula

$$
\begin{aligned}
& g=\sqrt{g_{x}^{2}+g_{y}^{2}} \\
& \theta=\arctan \frac{g_{y}}{g_{x}}
\end{aligned}
$$

where $\boldsymbol{g}$ is gradient magnitude, $\boldsymbol{\theta}$ is gradient orientation, $\boldsymbol{g}_{\boldsymbol{x}}$ is gradient in $x$ direction and $\boldsymbol{g}_{\boldsymbol{y}}$ is gradient in $y$ direction.

Then, cell histograms normalize to reduce the effect of changes in contrast between images of the 
same object [21].

\subsubsection{Hu moments}

The system uses Hu moments features for carpal region of interest (CROI). Moment invariants have been widely applied in image processing due to image translation, scaling and rotation invariants. It is a certain weighted average (moment) of the pixels' intensities in image processing. Moments can be applied in binary or grey scale images. Image' properties such as area, centroid and details about its orientation are found through image moments. Based on normalized central moments, $\mathrm{Hu}$ introduced moment invariants.

The Hu moments are calculated as follow:

$$
\begin{gathered}
I_{1}=\eta_{20}+\eta_{02} \\
I_{2}=\left(\eta_{20}-\eta_{02}\right)^{2}+4 \eta_{11}^{2} \\
I_{3}=\left(\eta_{30}-3 \eta_{12}\right)^{2}+\left(3 \eta_{21}-\eta_{03}\right)^{2} \\
I_{4}=\left(\eta_{30}+\eta_{12}\right)^{2}+\left(\eta_{21}+\eta_{03}\right)^{2} \\
I_{5}=\left(\eta_{30}-3 \eta_{12}\right)\left(\eta_{30}+\eta_{12}\right)\left[\left(\eta_{30}+\eta_{12}\right)^{2}-3\right. \\
\left.\left(\eta_{21}+\eta_{03}\right)^{2}\right]+\left(3 \eta_{21}-\eta_{03}\right)\left(\eta_{21}+\eta_{03}\right)\left[3 \left(\eta_{30}+\right.\right. \\
\left.\left.\eta_{12}\right)^{2}-\left(\eta_{21}+\eta_{03}\right)^{2}\right] \\
I_{6}=\left(\eta_{20}-\eta_{02}\right)\left[\left(\eta_{30}+\eta_{12}\right)^{2}-\left(\eta_{21}+\eta_{03}\right)^{2}\right]+4 \\
\eta_{11}\left(\eta_{30}+\eta_{12}\right)\left(\eta_{21}+\eta_{03}\right) \\
I_{7}=\left(3 \eta_{21}-\eta_{03}\right)\left(\eta_{30}+\eta_{12}\right)\left[\left(\eta_{30}+\right.\right. \\
\left.\left.\eta_{12}\right)^{2}-3\left(\eta_{21}+\eta_{03}\right)^{2}\right]-\left(\eta_{30}-3 \eta_{12}\right)\left(\eta_{21}+\eta_{03}\right) \\
{\left[3\left(\eta_{30}+\eta_{12}\right)^{2}-\left(\eta_{21}+\eta_{03}\right)^{2}\right]}
\end{gathered}
$$

Due to unchanged image rotation, translation, and scaling these moments are useful properties [22].

\subsection{Classification}

The proposed research applies Support Vector Machine (SVM) to classify bone age. SVM is a supervised machine learning algorithm to classify data. It constructs a hyperplane or set of hyperplanes in an infinite-dimensional space that can be used for regression and classification. It finds the hyperplane for classification which differentiates the two classes well. It is a binary classifier that create a model to classify new examples using a given training examples. A hyperplane separates the samples towards a maximum margin. SVM applies mathematical functions called kernel. The kernel function uses input data to transform a required form. SVMs apply different kinds of kernel functions.
There are different kinds of kernel functions such as linear, nonlinear, polynomial, radial basis function (RBF), and sigmoid, etc. The system uses SVM with polynomial kernel function. Polynomial kernel function can be calculated using the following equation

$$
k\left(x_{i} x_{j}\right)=\left(x_{i} x_{j}+1\right)^{d}
$$

where $\mathrm{d}$ is the degree of polynomial [23].

\section{Experimental results}

The dataset contains 1391 hand radiographs of children of age up to 18 years old by gender and race[9]. The proposed system used the dataset from University of Southern California. There are four races (ASI=Asian, BLK=African American, $\mathrm{CAU}=$ Caucasian and HIS=Hispanic) in the dataset. The experiment is tested EMROIs of middle finger and CROI of wrist hand by using 146 images of ASI and BLK females (110 training and 36 testing images) and 148 ASI, BLK males (112 training and 36 testing images) in age from one year to ten years old.

In this section, the system tests combination of HOG features from EMROIs and CROI, combination of $\mathrm{HOG}$ features from EMROIs and $\mathrm{Hu}$ moments features from CROI and then combination of HOG features from EMROIs and six geometric features from CROI. Geometric features are applied to describe the shape in this research. In this system, we apply six geometrical features such as perimeter, eccentricity, major axis length, minor axis length, area and extent of image' properties. The combination of $\mathrm{HOG}$ and $\mathrm{Hu}$ moments features achieved 5299 features and the using hog features achieved 7056 features. Then, using HOG features and six geometric features achieved 5298 features.

The performance of the proposed system is evaluated using Mean Absolute Error (MAE) and the Standard Deviation (SD) between the evaluations of the two radiologists and the computed bone age. MAE and SD values show the difference between the proposed methods and the two radiologists' evaluations. MAE and SD values is calculated using the following equations.

$$
\begin{aligned}
M A E & =\frac{\sum_{i=1}^{n}\left|y_{i}-x_{i}\right|}{n} \\
S D & =\sqrt{\frac{\sum|x-\mu|^{2}}{n}}
\end{aligned}
$$

where $y_{i}$ is age evaluated by radiologist 1 or 2 and $x_{i}$ is predicted age by proposed system. 
In the experiment of the system, the combination of $\mathrm{HOG}$ and $\mathrm{Hu}$ moments method obtains 1.2717 years of mean absolute error (MAE) compared with radiologist 1, 1.1997 years of (MAE) compared with radiologist 2 in ASI, BLK females and 1.3681 years (MAE) compared with radiologist 1, 1.3311 years compared with radiologist 2 in ASI, BLK males. Fig. 5 and Fig. 6 show the comparison between the

Table 1. MAE and SD values of ASI, BLK males

\begin{tabular}{|l|l|l|l|l|l|l|}
\hline & \multicolumn{3}{|l|}{ Radiologist 1 } & \multicolumn{3}{l|}{ Radiologist 2 } \\
\hline & $\begin{array}{l}\text { HO } \\
\text { G }\end{array}$ & $\begin{array}{l}\text { HO } \\
\text { G+ } \\
\text { Hu }\end{array}$ & $\begin{array}{l}\text { HOG } \\
\text { +Geo }\end{array}$ & HOG & $\begin{array}{l}\text { HO } \\
\text { G+ } \\
\text { Hu }\end{array}$ & $\begin{array}{l}\text { HOG+ } \\
\text { Geo }\end{array}$ \\
\hline $\begin{array}{l}\text { M } \\
\text { AE }\end{array}$ & 1.31 & 1.36 & 1.39 & 1.28 & 1.33 & 1.42 \\
\hline SD & 1.85 & 1.95 & 2.26 & 1.86 & 1.95 & 2.30 \\
\hline
\end{tabular}

Table 2. MAE and SD values of ASI, BLK females

\begin{tabular}{|l|l|l|l|l|l|l|}
\hline & \multicolumn{3}{|l|}{ Radiologist 1 } & \multicolumn{3}{l|}{ Radiologist 2 } \\
\hline & $\begin{array}{l}\text { HO } \\
\text { G }\end{array}$ & $\begin{array}{l}\text { HO } \\
\text { G+H } \\
\text { u }\end{array}$ & $\begin{array}{l}\text { HOG } \\
+ \text { Geo }\end{array}$ & HOG & $\begin{array}{l}\text { HOG } \\
+ \text { Hu }\end{array}$ & $\begin{array}{l}\text { HOG } \\
+ \text { Geo }\end{array}$ \\
\hline $\begin{array}{l}\text { M } \\
\text { AE }\end{array}$ & 1.23 & 1.27 & 1.69 & 1.195 & 1.199 & 1.57 \\
\hline SD & 1.66 & 1.80 & 1.91 & 1.63 & 1.77 & 1.87 \\
\hline
\end{tabular}

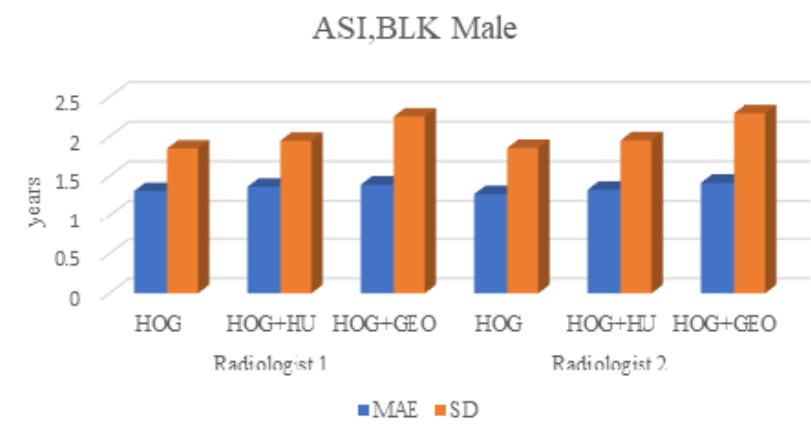

Figure. 5 MAE and SD values of the proposed methods in ASI, BLK males

\section{ASI, BLK Females}

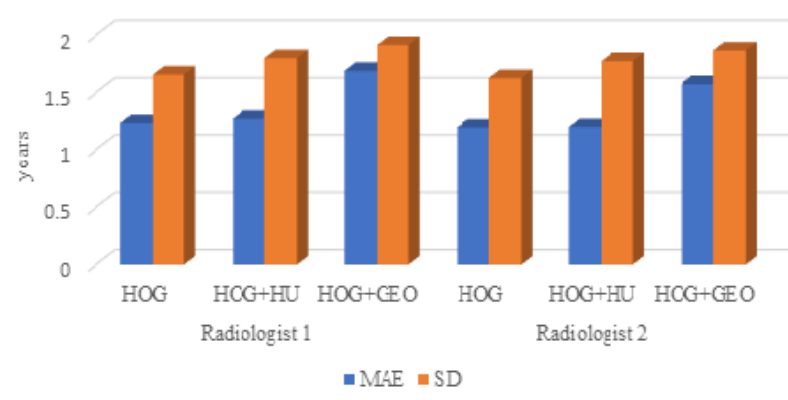

Figure. $6 \mathrm{MAE}$ and SD values of the proposed methods in ASI, BLK females proposed methods with MAE and SD values from age range (1 to 10 years) for ASI, BLK female and male radiographs. Table 1 and 2 show the results of MAE and SD values on testing EMROIs and CROI of ASI and BLK races in age 1 to 10 years by comparing the evaluations of radiologist 1 and radiologist 2 .

In Table 3, combination of HOG from EMROIs and CROI obtains 7056 features, combination of HOG and Hu moments features obtains 5299 features and combination of HOG and six geometric features obtains 5298 features. According to Table 3, the combination of $\mathrm{HOG}$ and $\mathrm{Hu}$ moments method achieves accuracy $89 \%$ in ASI, BLK females and $86 \%$ in ASI, BLK males. The combination of $\mathrm{HOG}$ features from EMROIs and CROI achieved performance accuracy $88 \%$ in ASI, BLK females and $84 \%$ in ASI, BLK males. Then, the combination HOG features from EMROIs and six geometrical features from CROI achieved performance accuracy $85 \%$ in ASI, BLK females and $85 \%$ in ASI, BLK males. Combination of $\mathrm{HOG}$ and $\mathrm{Hu}$ moments features reduces feature dimensions. By comparing

Table 3. Evaluation results of ASI, BLK races from age 1

\begin{tabular}{|l|l|l|l|}
\hline Method & $\begin{array}{l}\text { Number } \\
\text { of } \\
\text { Features }\end{array}$ & $\begin{array}{l}\text { Accuracy } \\
\text { (Female) }\end{array}$ & $\begin{array}{l}\text { Accuracy } \\
\text { (Male) }\end{array}$ \\
\hline HOG & 7056 & $88 \%$ & $84 \%$ \\
\hline HOG+Hu & 5299 & $89 \%$ & $86 \%$ \\
\hline HOG+Geometric & 5298 & $85 \%$ & $85 \%$ \\
\hline
\end{tabular}

Table 4. Analysis of execution time for ASI, BLK races

\begin{tabular}{|c|c|c|c|c|c|c|}
\hline \multirow[b]{2}{*}{$\begin{array}{l}\text { Met } \\
\text {-hod }\end{array}$} & \multicolumn{3}{|c|}{ Time(sec) for Male } & \multicolumn{3}{|c|}{ Time(sec) for Female } \\
\hline & $\begin{array}{l}\text { Feat } \\
\text {-ure } \\
\text { extr- } \\
\text { actio } \\
\text {-n }\end{array}$ & $\begin{array}{l}\text { Trai } \\
\text {-ing } \\
\text { Tim } \\
\text {-e }\end{array}$ & $\begin{array}{l}\text { Test } \\
\text {-ing } \\
\text { Tim } \\
\text { e }\end{array}$ & $\begin{array}{l}\text { Featu } \\
\text {-re } \\
\text { extra } \\
\text { ction }\end{array}$ & $\begin{array}{l}\text { Train } \\
\text {-ing } \\
\text { Time }\end{array}$ & $\begin{array}{l}\text { Test } \\
\text {-ing } \\
\text { Tim } \\
\text { e }\end{array}$ \\
\hline $\begin{array}{l}\text { HO } \\
\text { G }\end{array}$ & 0.01 & 5.80 & 1.86 & 0.002 & 5.76 & 1.86 \\
\hline $\begin{array}{l}\text { HO } \\
\text { G+ } \\
\text { Hu }\end{array}$ & 0.01 & 5.60 & 1.71 & 0.002 & 5.59 & 1.59 \\
\hline $\begin{array}{l}\text { HO } \\
\text { G+ } \\
\text { Geo }\end{array}$ & 0.02 & $\begin{array}{l}43.3 \\
3\end{array}$ & 2.29 & 0.18 & 28.57 & 1.91 \\
\hline
\end{tabular}

Table 5. Comparison of performance between the proposed method and previous works

\begin{tabular}{|l|l|l|}
\hline Methods & $\begin{array}{l}\text { Accuracy } \\
\text { of Male }\end{array}$ & $\begin{array}{l}\text { Accuracy } \\
\text { of Female }\end{array}$ \\
\hline$[17]$ & $80.4 \%$ & $83.3 \%$ \\
\hline$[16]$ & $61.40 \%$ & $57.32 \%$ \\
\hline$[14]$ & $68.63 \%$ & $73.88 \%$ \\
\hline Proposed Method & $86 \%$ & $89 \%$ \\
\hline
\end{tabular}

DOI: $10.22266 /$ ijies2021.0630.11 
these methods, combining Hog features from EMROIs and $\mathrm{Hu}$ moments features from CROI achieved better results than the other methods. Table 4 shows the analysis of feature extraction time, training time and testing time for ASI, BLK races in age 1 to 10 years old.

Table 5 shows the performance of the proposed system and other previous works. As shown in Table 5 , the proposed method outperformed to other methods in accuracy.

\section{Conclusion}

The proposed research presents an automated skeletal BAA method that implements middle finger bones and carpal bones of hand wrist. The system assesses bone age estimation method by integrating both EMROIs and CROI which is reliable and accurate for BAA. The system applies SVM with polynomial kernel function to classify bone age. The proposed research achieves the accuracy of $89 \%$ of ASI, BLK females and $86 \%$ of ASI, BLK males in testing HOG features from EMROIs of middle finger and $\mathrm{Hu}$ moments features from CROI of carpal bones from age one year to 10 years old. Combining HOG features from EMROIs and $\mathrm{Hu}$ moments features from CROI achieves better results than the other methods. In future work, the system will test the other Caucasian (CAU) and Hispanic (HIS) races in the dataset.

\section{Conflicts of Interest}

The authors declare no conflict of interest with respect to the research, authorship, and/or publication of this article.

\section{Author Contributions}

Aye Aye Aung contributed to the design and implementation of the research, the analysis of the results, and the writing of the manuscript. Dr. Zin Mar Win supervised this research work.

\section{References}

[1] P. Thangam, T. V. Mahendiran, EEE Department, Coimbatore Institute of Engineering and Technology, Coimbatore 641109, Tamilnadu, India, K. Thanushkodi, and Akshaya College of Engineering and Technology, Coimbatore - 642109, Tamilnadu, India, "Skeletal Bone Age Assessment Research Directions", J. Eng. Sci. Technol. Rev., Vol. 5, No. 1, pp. 90-96, 2012, doi: 10.25103/jestr.051.16.
[2] D. Giordano, C. Spampinato, G. Scarciofalo, and R. Leonardi, "Automatic skeletal bone age assessment by integrating EMROI and CROI processing", In: Proc. of 2009 IEEE International Workshop on Medical Measurements and Applications, Cetraro, pp. 141-145, 2009, doi: 10.1109/MEMEA.2009.5167972.

[3] M. Satoh, "Bone age: assessment methods and clinical applications", Clin. Pediatr. Endocrinol., Vol. 24, No. 4, pp. 143-152, 2015, doi: 10.1297/cpe.24.143.

[4] A. M. Mughal, N. Hassan, and A. Ahmed, "Bone Age Assessment Methods: A Critical Review," Pak. J. Med. Sci., Vol. 30, No. 1, 1969, doi: 10.12669/pjms.301.4295.

[5] A. Schmeling, G. Geserick, W. Reisinger, and A. Olze, "Age estimation", Forensic Sci. Int., Vol. 165, No. 2-3, pp. 178-181, 2007, doi: 10.1016/j.forsciint.2006.05.016.

[6] R. P. Spencer, S. Sami, M. Karimeddini, J. J. Sziklas, and R. Rosenberg, "Role of bone scans in assessment of skeletal age", Int. J. Nucl. Med. Biol., Vol. 8, No. 1, pp. 33-38, 1981, doi: 10.1016/0047-0740(81)90051-6.

[7] E. Pietka, L. Kaabi, M. L. Kuo, and H. K. Huang, "Feature extraction in carpal-bone analysis", IEEE Trans. Med. Imaging, Vol. 12, No. 1, pp. 44-49, 1993, doi: 10.1109/42.222665.

[8] G. K. Manos, A. Y. Cairns, I. W. Rickets, and D. Sinclair, "Segmenting radiographs of the hand and wrist", Comput. Methods Programs Biomed., Vol. 43, No. 3-4, pp. 227-237, 1994, doi: 10.1016/0169-2607(94)90074-4.

[9] A. Gertych, A. Zhang, J. Sayre, S. PospiechKurkowska, and H. K. Huang, "Bone age assessment of children using a digital hand atlas", Comput. Med. Imaging Graph., Vol. 31, No. 45, pp. 322-331, 2007, doi: 10.1016/j.compmedimag.2007.02.012.

[10] J. Liu, J. Qi, Z. Liu, Q. Ning, and X. Luo, "Automatic bone age assessment based on intelligent algorithms and comparison with TW3 method", Comput. Med. Imaging Graph., Vol. 32, No. 8, pp. 678-684, 2008, doi: 10.1016/j.compmedimag.2008.08.005.

[11] J. Seok, B. Hyun, J. Kasa-Vubu, and A. Girard, "Automated classification system for Bone Age X-ray images", In: Proc. of 2012 IEEE International Conf. on Systems, Man, and Cybernetics (SMC), Seoul, Korea (South), pp. 208-213, 2012, doi: 10.1109/ICSMC.2012.6377701.

[12] G. E. G. Raksin and H. Ug, "Bone age determination in young children (newborn to 6 
years old) using support vector machines", Turkish Journal of Electrical Engineering and Computer Sciences, Vol. 24, No. 3, pp. 16931708, 2016.

[13] J.-A. Choi, Y. C. Kim, S. J. Min, and E. K. Khil, "A simple method for bone age assessment: the capitohamate planimetry", Eur. Radiol., Vol. 28, No. 6, pp. 2299-2307, 2018, doi: 10.1007/s00330-017-5255-4.

[14] F. Dehghani, A. Karimian, and M. Sirous, "Assessing the Bone Age of Children in an Automatic Manner Newborn to 18 Years Range", J. Digit. Imaging, Vol. 33, No. 2, pp. 399-407, 2020, doi: 10.1007/s10278-01900209-z.

[15] D. Wang, K. Zhang, J. Ding, and L. Wang, "Improve bone age assessment by learning from anatomical local regions", ArXiv200513452 Cs, 2020, Accessed: Jan. 17, 2021. [Online]. Available: http://arxiv.org/abs/2005.13452.

[16] H. Lee, S. Tajmir, J. Lee, M. Zissen, B. A. Yeshiwas, T. K. Alkasab, G. Choy, and S. Do, "Fully Automated Deep Learning System for Bone Age Assessment", J. Digit. Imaging, Vol. 30, No. 4, pp. 427-441, 2017, doi: 10.1007/s10278-017-9955-8.

[17] V. Pinchi, F. D. Luca, M. Focardi, F. Pradella, G. Vitale, F. Ricciardi and G. A. Norelli, "Combining dental and skeletal evidence in age classification: Pilot study in a sample of Italian sub-adults", Leg. Med., Vol. 20, pp. 75-79, 2016, doi: 10.1016/j.legalmed.2016.04.009.

[18] P. Perona and J. Malik, "Scale-space and edge detection using anisotropic diffusion", IEEE Trans. Pattern Anal. Mach. Intell., Vol. 12, No. 7, pp. 629-639, 1990, doi: 10.1109/34.56205.

[19] D. Giordano, C. Spampinato, G. Scarciofalo, and R. Leonardi, "An Automatic System for Skeletal Bone Age Measurement by Robust Processing of Carpal and Epiphysial/Metaphysial Bones", IEEE Trans. Instrum. Meas., Vol. 59, No. 10, pp. 2539-2553, 2010, doi: 10.1109/TIM.2010.2058210.

[20] X. Bai, F. Zhou, and B. Xue, "Image enhancement using multi scale image features extracted by top-hat transform", Opt. Laser Technol., Vol. 44, No. 2, pp. 328-336, 2012, doi: 10.1016/j.optlastec.2011.07.009.

[21] S. Mallick, "Histogram of Oriented Gradients", p. 18.

[22] Zhihu Huang and Jinsong Leng, "Analysis of $\mathrm{Hu}$ 's moment invariants on image scaling and rotation", In: Proc. of 2010 2nd International Conf. on Computer Engineering and Technology,
Chengdu, China, 2010, pp. V7-476-V7-480, doi: 10.1109/ICCET.2010.5485542.

[23] C. Cortes and V. Vapnik, "Support-vector networks", Mach. Learn., Vol. 20, No. 3, pp. 273-297, 1995, doi: 10.1007/BF00994018. 